\title{
COYOTE (Canis latrans) HABITAT USE AND MORTALITY IN Grand Teton National Park and SubUrban- Agricultural Areas of Jackson Hole, Wyoming
}

\author{
MATT MCGEE $\uparrow$ STAN ANDERSON \\ WYOMING COOPERATIVE FISH AND WILDLIFE RESEARCH UNIT \\ DOUG WACHOB $\uparrow$ TETON SCIENCE SCHOOL $\uparrow$ KELLY, WY
}

\section{$\downarrow$ ABSTRACT}

A study of coyote (Canis latrans) habitat use and mortality in Grand Teton National Park and the suburban-agricultural land surrounding Jackson, WY was conducted between September 1999 and August 2000. This research focused on the influence of human development, habitat type, topography, and simulated wolf presence on coyote habitat use and on coyote mortality patterns in undeveloped and suburban-agricultural land. The overall goal of this project was to provide baseline information on the coyote population in Jackson Hole that can be used in the future to determine what, if any, impact wolves and human developments may have on coyotes. There were a total of fifteen radio-collared coyotes in the suburban-agricultural area and fourteen radio collared coyotes in Grand Teton National Park and adjacent areas in the National Elk Refuge and Bridger-Teton National Forest. Marked coyotes were tracked weekly using short interval telemetry relocations and triangulation to determine habitat use patterns. During the winter, track transects were skied weekly and coyote trails were backtracked and mapped using hand held GPS units to determine fine scale habitat use patterns. Coyote mortality was determined via telemetry and direct observation. Preliminary data analyses suggest that coyotes use mainly sagebrush-grasslands or forest-shrub-grass edge areas and avoid forest interior areas. Coyotes frequently use trails and roads in the undeveloped area when moving long distances. Preliminary analysis also indicates that roads and trails are used in
\end{abstract}

a greater proportion than their abundance on the landscape. Coyotes were frequently observed using riparian corridors to move between open meadows in the suburban-agricultural area. There is some evidence that suggests coyotes selectively travel fences and irrigation ditches for long distances in agricultural areas. The movement data also suggests that coyotes avoid developed areas during the day and travel in these developed areas at night. The data on coyote locations suggests some avoidance of wolf urine scent grids in the undeveloped area, but not in the developed area. Coyote mortality was primarily human caused, and coyotes that were male, transient, and lived in the suburban-agricultural area were the most commonly killed animals.

\section{$\uparrow \quad$ INTRODUCTION}

Coyotes (Canis latrans) are an abundant predator widely distributed in North America because of their generalist diet, flexible social organization, and diverse habitat use (Bowen 1981, Messier and Barrette 1982). They originally occupied grassland habitats in the western United States and have adapted to live in a diverse mix of habitats, including desert, forest, agricultural, and urban areas in the past century (Moore and Parker 1992, Sheldon 1992). The decrease in the abundance and distribution of wolves as well as the increase in human developments has directly influenced the expansion of coyotes from the western United States throughout North and Central America (Gier 1975, Nowak 1978, 
Bounds 1994). Decreased interference competition from wolves has allowed coyotes to occupy new territories, and human developments provide a new source of food in the form of garbage, pets, pet food, and livestock. Habitat alteration from clear cuts and fragmentation of habitat may also increase the food available to coyotes (Toweil and Anthony 1988).

This project, combined with the research of McClennen (2000) and Wigglesworth (2000), provides baseline information on the coyote population in Jackson Hole. Information on how coyotes use ranchland and suburban habitat types in Jackson Hole may be useful for future planning in Teton County. This county has the fastest growing population in Wyoming with a $61 \%$ increase from 1990 to 2000 (U.S. Census Bureau 2000). This information on coyote habitat use may also be useful in developing management strategies to deal with human-coyote conflicts that are reasonable both in conserving coyotes and protecting human interests. Information on the baseline parameters of the coyote population in Jackson Hole will also be useful for measuring the impact of wolf re-colonization on coyotes in the future. Wolf re-colonization is a rare ecological event that may have significant impacts on the established coyote population in Jackson Hole. Wolves have been shown to influence coyote habitat use in Isle Royale National Park, MI (Mech 1966), Northern Minnesota (Berg and Chesness 1978), Riding Mountain National Park, Alberta (Carbyn 1982), and Yellowstone National Park, WY (Crabtree and Sheldon 1999).

There have been seven significant studies conducted on coyotes in Jackson Hole. These studies include an extensive investigation of the stomach contents of coyotes (Murie 1935). Weaver (1977) compared coyote densities to prey availability in three different locations. Camenzind (1978) and Bekoff and Wells (1980) both investigated coyote social behavior and organization in the National Elk Refuge and Grand Teton National Park. Tzilkowski (1980) investigated mortality patterns of coyotes in Grand Teton National Park. McClennen (2000) investigated the effects of suburban and agricultural development on coyote home range size, activity patterns, movement, and density. Wigglesworth (2000) investigated coyote habitat use, diet, social organization, and disease seroprevalence in Grand Teton National Park and suburban and agricultural areas. This project investigates the influence of human development, habitat type, topography, and simulated wolf presence on fine scale movements and home range scale coyote habitat use prior to extensive colonization of the Jackson Hole area by wolves. This project also investigates the causes of mortality for marked coyotes, and the habitat use, gender, social status, and activity cycles of coyotes that died during the study.

\section{METHODS}

\section{Study Area}

The valley of Jackson Hole $\left(43^{\circ} 40^{\prime}\right.$ latitude, $110^{\circ} 43^{\prime}$ longitude) was divided into two study areas: the undeveloped area (UNDA) and the suburban-agricultural area (SAA). These areas were selected primarily because they represent a gradient of human land use practices including protected public land and private agricultural-suburban land. This area presents an interesting location in which to study both the influence of human development and wolves on coyote habitat use and mortality patterns. The distribution of land use along with the presence of a coyote population that has lived in the absence of wolves for the last sixty years, and the current wolf re-colonization combine to create a unique research opportunity.

The UNDA includes parts of southeastern Grand Teton National Park (GTNP), the National Elk Refuge (NER), and the western edge of BridgerTeton National Forest (BTNF) adjacent to GTNP. This area has a relatively low density of housing, ranging from $0-0.08$ per hectare in the sections used by coyotes and has a lower density of roads (3.9 $\mathrm{km} / \mathrm{ha}$ ) than the SAA. Kelly, WY (Pop. 200), the Gros Ventre Campground, and a few private inholdings are located in the UNDA. There is a limited amount of cattle grazing on specified allotments during the summer in GTNP. It is illegal to kill coyotes in GTNP and the NER, but not in BTNF. In the UNDA the valley is primarily vegetated with sagebrush (Artemesia spp.) and patches of grasses in old hayfields and burned areas. The hillsides are primarily vegetated by mixed conifer stands including Douglas-fir (Pseudotsuga menziesii), lodgepole pine (Pinus contorta), and subalpine fir (Abies lasiocarpa) interspersed with smaller aspen (Populus tremuloides) stands. The Gros Ventre and Snake River riparian areas are primarily vegetated by narrowleaf cottonwood (Populus angustofolium), Colorado blue spruce (Picea pungens), and willow (Salix spp.).

The SAA is centered on the towns of Jackson and Wilson and encompasses mainly agricultural land interspersed with rapidly growing 
suburban development along the Snake River corridor. It extends from the south boundary of Grand Teton National Park to the junction of the Snake River and Highway 191. The SAA has a relatively high density of housing, ranging from 0.03 0.99 houses per hectare and a higher density of roads $(17.3 \mathrm{~km} / \mathrm{ha})$ than the UNDA. There is abundant cattle ranching, and it is legal to kill coyotes in most parts of this area unless otherwise prohibited by city ordinances. In the SAA the valley is primarily vegetated with grasses in hayfields interspersed with sagebrush, and developed areas. Mixed conifer and aspen stands exist primarily on the hillsides throughout the SAA. The Snake River riparian area in the SAA has similar vegetation characteristics to the riparian areas in the UNDA. The riparian area in the SAA however makes up a larger proportion of the study area relative to the riparian area in the UNDA.

\section{$\uparrow \quad$ METHODS}

The general data collection and analysis methods of this study follows those used by McClennen (2000) and Wigglesworth (2000) in order to allow comparison of data sets with their research. The field portion of this study was conducted between September 1999 and August 2000. Data collection was divided into three seasons based on current knowledge of coyote biology: 1) pair formation-gestation (1 January- 30 April), pup rearing (1 May- 31 July), and dispersal (1 August- 31 December) (Smith et al. 1981). Coyote movement patterns were compared with vegetation layers created on a Geographic Information System (GIS) using ArcInfo and ArcView software (ESRI, Redlands, CA). Vegetation layers were modified from a landcover data set derived from LANDSAT thematic mapper (TM) imagery with $30 \mathrm{~m}$ pixel resolution (Idaho/Western Wyoming Landcover Classification Report and Metadata, Logan UT 1995). Vegetation coverages (GIS layers) from GTNP, the NER, and aerial photos (Teton County 1999) were used to correct the TM coverage.

During October 1999, seven new coyotes were trapped and collared in the developed study area and one new coyote was trapped and collared in the undeveloped study area to increase the sample size to twenty-nine collared animals (15 in the developed area, 14 in the undeveloped area). Coyotes were trapped using \#3 Softcatch padded leghold traps (Woodstream Corp, Lititz, PA). Traps were checked twice every 24 hours throughout the capture periods. Captured coyotes weighing 7.5-16 kg were given ketamine hydrochloride $(4.7 \mathrm{mg} / \mathrm{kg})$ and xilazine hydrochloride $(1.6 \mathrm{mg} / \mathrm{kg})$ in order to anesthetize and sedate them during handling (Servin and Huxley 1992). Yohimbine $(0.15 \mathrm{mg} / \mathrm{kg})$ was used to reverse the effects of the ketamine (Kreeger 1992). Coyotes were fitted with radio collars equipped with activity and mortality signals (Advanced Telemetry Systems (ATS), Isanti, MN), sexed, weighed, and aged by tooth wear (Gier 1968). Blood samples were taken for disease seroprevalence research (Wigglesworth 2000). Radio telemetry was used throughout the study to locate coyotes. Telemetry locations were determined using two truck mounted null-peak antenna systems, fixed wing aircraft telemetry, and hand held antenna systems. Telemetry surveys were conducted to locate all coyotes in each study area twice a week during each biological season. Telemetry data collection was divided into four 6 hour time blocks to allow for equal sampling at all times of the day and night (6:00-12:00, 12:00-18:00, 18:00-24:00, 24:00-6:00). The sampling time period used each week was randomly selected. For analysis, coyotes were classified into social types as either transients or residents (Gese et al. 1988).

Coyote movement patterns (travel paths) were determined using two truck mounted null-peak antenna systems and a portable null-peak antenna tower (ATS, Isanti, MN). Sequential simultaneous azimuths were taken every 15 minutes from known locations to track coyote movement (Laundre and Keller 1981). Randomly selected coyotes were tracked during 6-hour telemetry sessions, which rotated around a 24-hour day so that coyote travel was determined in both day and night hours. Equal sampling efforts were attempted in each study area for all collared animals approximately weekly from 1 January 2000 to 1 August 2000. Locations were calculated using the program LOCATE II (Pacer, Truro, Nova Scotia, Canada) on HP200LX palmtop computers (Hewlett-Packard Co., Dallas, TX).

Coyote movement patterns were also determined from snow tracking transects. Data was collected from five transects in each study area that measured approximately $2.5 \mathrm{~km}$ in length. These transects were located on closed roads or trails within known coyote home ranges to increase the probability of tracking marked animals in resident groups. The location of each transect was not selected randomly since the goal was to increase the chance of tracking marked animals within known coyote home ranges. Each transect was skied or snow shoed once a week preferably 24 to 48 hours after new snowfall from 1 December 1999 to 1 April 2000. Any coyote tracks that intersected the transect 
were backtracked as far as possible and point locations along the coyote tracks were determined every 200 meters using a hand held global positioning system (GPS) unit (Garmin 12xl, Garmin Inc Olathe, KS). The accuracy of these locations was within approximately 100 meters.

Coyote mortality was determined using telemetry information, carcasses, and direct observations. In the case of mortalities in the marked sample the carcasses were recovered if possible, and sent to the Wyoming Veterinary Lab for a necropsy. If there was no carcass, the cause of death was determined from any witnesses or persons involved.

Wolf urine scent stations were established in each study area during the pair formation-gestation (1 January - 30 April) and pup rearing (1 May- 31 July) biological seasons. These scent stations were placed in the approximate center of selected coyote home ranges calculated by data from McClennen (pers. comm.). Five coyote territories were used as experimental sites with wolf urine scent stations and an equal number were used as control sites without wolf urine scent stations. The scent stations were made up of a 10 by 10 meter grid with wolf urine scent placed every 5 meters. Wolf urine $(10 \mathrm{ml})$ was placed on vegetation or on open ground within the grid. These stations were re-scented every week during this phase of the study. In winter scent stakes were used in order to prevent scent from being buried by snow. The control sites were visited every week and the grid walked without placing any scent. Coyote locations were monitored weekly using radio telemetry to determine any shifts in habitat use patterns relative to the wolf urine scent grids.

\section{$\downarrow \quad$ RESUlTS AND DISCUSSION}

\section{Habitat Use}

In the SAA data on 134 coyote travel paths was collected, and in the UNDA data on 162 travel paths was collected from snow tracking during the period from 1 Dec 1999 to 1 April 2000. The travel paths range in length from 200 meters to $12 \mathrm{~km}$. Preliminary data analyses comparing coyote habitat use to the total available habitat in the SAA suggests that coyotes in the SAA use mainly aspen, grasslands, and riparian habitat or forest-grass edge areas and avoid forest interior areas. Aspen, grass and riparian habitat types were used in slightly greater proportion to their availability. Shrub and developed habitat types were used in lesser proportion to their availability. In addition conifer habitat was used in approximate proportion to its availability (Table 1). Since the bare ground habitat classification made up only one percent of the study area it was not included in this analysis. These results indicate that with the exception of avoiding developed areas coyote habitat use is influenced by factors other than just habitat type e.g. territoriality or prey availability. Analyses were also conducted comparing coyote habitat use to the available habitat in buffered areas with a radius equal to the half the total distance from the initial point to the farthest point in each travel path (Table 2). Preliminary analysis also indicates that roads and trails were used in a greater frequency than their abundance on the landscape. Coyotes also frequently used riparian corridors to travel between open meadows in the SAA. In addition there is some evidence that suggests coyotes selectively travel fences and irrigation ditches for long distances in agricultural areas.

Table 1: Average \% use by habitat type for coyote travel path data from snow tracking in the SAA compared to total available habitat in the study area

\begin{tabular}{|c|c|c|c|c|c|c|}
\hline SAA-Summary 1 & A spen & $\underline{\text { Shrub }}$ & Conifer & Grass & Riparian & Developed \\
\hline$\%$ Used & $1.64 \%$ & $9.71 \%$ & $8.97 \%$ & $53.18 \%$ & $25.29 \%$ & $0.69 \%$ \\
\hline $\begin{array}{l}\% \text { Available } \\
\text { in SAA } \\
\text { Ratio }\end{array}$ & $1.22 \%$ & $\% 11.20$ & $8.90 \%$ & $42.93 \%$ & $22.39 \%$ & $11.51 \%$ \\
\hline Used/Available & 1.34 & 0.87 & 1.01 & 1.24 & 1.13 & 0.06 \\
\hline
\end{tabular}

Table 2: Average \% use by habitat type for coyote travel path data from snow tracking in the SAA compared to average available habitat in buffered areas with a radius equal to the half the total distance from the initial point to the farthest point in each path

\begin{tabular}{|c|c|c|c|c|c|c|}
\hline SAA-Summary 2 & A spen & Shrub & Conifer & Grass & Riparian & Developed \\
\hline $\begin{array}{c}\% \text { Used } \\
\% \text { Available in }\end{array}$ & $1.64 \%$ & $9.71 \%$ & $8.97 \%$ & $53.18 \%$ & $25.29 \%$ & $0.69 \%$ \\
\hline $\begin{array}{c}\text { Buffers } \\
\text { Ratio }\end{array}$ & $2.63 \%$ & $10.58 \%$ & $8.70 \%$ & $51.56 \%$ & $23.46 \%$ & $2.04 \%$ \\
\hline Used/Available & $0.62^{*}$ & 0.92 & 1.03 & 1.03 & 1.08 & 0.34 \\
\hline
\end{tabular}

Preliminary data analyses suggest that coyotes in the UNDA use mainly shrub and grassland habitats and forest-grass/shrub edge areas and avoided forest interior areas. Shrub and grass habitat types were used in greater proportion to their availability, and aspen, conifer and riparian habitat types were used in lesser proportion to their availability (Table 3 ). Analyses were also conducted comparing coyote habitat use to the available habitat in buffered areas with a radius equal to the half the total distance from the initial point to the farthest 
point in each travel path (Table 4). Since the bare ground and developed habitat classifications made up less than one percent of the study area they were not included in this analysis. Preliminary analysis also indicates that roads and trails were used in a greater frequency than their abundance on the landscape in the UNDA. There is some evidence that suggests that coyotes selectively travel along old irrigation ditches in the Kelly hayfields area.

Table 3: Average \% use by habitat type for coyote travel path data from snow tracking in the UNDA compared to total available habitat in the study area

\begin{tabular}{|ccccccc|}
\hline UNDA-Summary 1 & $\underline{\text { Aspen }}$ & $\underline{\text { Shrub }}$ & & Conifer & Grass & Riparian \\
\% Used & $4.04 \%$ & $53.47 \%$ & $5.80 \%$ & $32.62 \%$ & $3.86 \%$ \\
\% Available in UNDA & $5.22 \%$ & $37.31 \%$ & $34.72 \%$ & $16.79 \%$ & $5.48 \%$ \\
& & & & & \\
Ratio Used/Available & $\mathbf{0 . 7 7}$ & $\mathbf{1 . 4 3}$ & $\mathbf{0 . 1 7}$ & $\mathbf{1 . 9 4}$ & $\mathbf{0 . 7}$ \\
\hline
\end{tabular}

Table 4: Average $\%$ use by habitat type for coyote travel path data from snow tracking in the UNDA compared to average available habitat in buffered areas with a radius equal to the half the total distance from the initial point to the farthest point in each path

\begin{tabular}{|c|c|c|c|c|c|}
\hline UNDA-Summary 2 & Aspen & $\underline{\text { Shrub }}$ & Conifer & $\underline{\text { Grass }}$ & $\underline{\text { Riparian }}$ \\
\hline$\%$ Used & $4.04 \%$ & $53.47 \%$ & $5.80 \%$ & $32.62 \%$ & $3.86 \%$ \\
\hline$\%$ Available in Buffers & $3.82 \%$ & $45.03 \%$ & $10.48 \%$ & $35.24 \%$ & $4.83 \%$ \\
\hline Ratio Used/Available & $1.06^{*}$ & 1.19 & 0.55 & $0.93^{*}$ & 0.80 \\
\hline
\end{tabular}

* Indicates different result from Table 3

Preliminary results suggest that the nonrandom location of the track transects in known coyote home ranges mainly in and around grasslands and riparian areas in the SAA and shrub and grasslands in the UNDA may have influenced the results of the data. This could have biased the results toward grass, shrub, and riparian habitat types and away from forest and developed habitat types. Additionally, natural and human caused disturbances to the snow could have resulted in reduced detection of coyote tracks, and increased habitat biases. For example wind, snowfall, skiers, and snowplows reduced the ability to detect coyote tracks in some habitats more than in others. Determining GPS locations could have also been influenced by habitat based biases in that the ability to get accurate GPS locations varies in relation to habitat type and may be more accurate in some habitats than in others. Correction of this bias was attempted by analyzing the habitat used in each travel path relative to a buffered area around a point location centered on the mid point of the distance between the initial point and the farthest point in each travel path. This buffered area had a radius equal to half the total distance from the initial point to the farthest point (Table 2 and Table 4). This effectively limits the available habitat for comparison to a subset of the total study area that is in close proximity to the travel path. Results from this method were similar in all cases in the SAA except for the aspen habitat type that appears to be used in greater proportion to its availability when investigating use vs. total available habitat in the SAA (Table 1). It appears however, to be underused when investigating use vs. available habitat in travel path buffers (Table 2). There were two cases in which the results of the use vs. buffered availability analysis were different from the use vs. total availability analysis in the UNDA. Aspen habitats appear to be underused when investigating use vs. total available habitat in the UNDA (Table 3), however it appears to be used in approximate proportion to its availability when investigating the use vs. availability in travel path buffers (Table 4). Grass habitat appears to be used in greater proportion to its availability when investigating use vs. total available habitat in the UNDA (Table 3), however it appears to be used in approximate proportion to its availability when investigating the use vs. availability in travel path buffers (Table 4). These results suggest that factors other than habitat type (territory competition or prey availability) may have a more significant influence on coyote habitat use in this population.

Further research should attempt to develop a stratified random sampling method that may remove any habitat based bias from the transect locations by sampling all available habitat types approximately equally. Further research should also attempt to account for any inherent habitat bias present due to the variable detection of tracks between habitat types.

Data analysis on a total of 118 coyote travel paths in the UNDA and 115 coyote travel paths in the SAA were conducted using 15-minute interval sequential locations. The quality of the resolution of these movement patterns is highly variable depending on the area of the valley sampled. This variability indicates a potential habitat based bias in which certain habitats were easier to track animals in than other habitats. This type of bias will commonly overestimate the use of habitats in which it is easier to detect coyotes and underestimate habitats in which it is difficult to track coyotes. However, some of the travel paths suggest relatively clear movement patterns. Preliminary analyses indicate that some marked animals frequently used the same travel paths when traveling within the core area of their known 
home range. These frequently used "travel corridors" were often associated with trails and roads, and prominent topographic features (creeks, ridges, ditches). There are some patterns similar to those observed in the snow tracking data that suggests open sagebrush grasslands or forest shrub edge areas may be used more frequently than their availability, and that riparian corridors are a frequently used travel corridor especially in the SAA. The movement data also suggests that coyotes avoid developed areas during the day and travel in these developed areas at night especially in the SAA.

Further research should make use of portable null peak towers or fixed towers at high points in the valley (Blacktail Butte, Miller Butte, Shadow Mt. or Signal Mt.) in order to improve the quality of the locations, minimize any habitat based bias, and increase the time that it is possible to track individual animals. It would also be worth experimenting $w$ ith $u$ sing less frequent triangulation (e.g. every hour) to investigate movement patterns on a larger time scale than the short interval relocations attempt. For example, continuous monitoring every hour for 24 hours may produce interesting results on high activity areas, and suggest behaviors associated with movement patterns in these areas (Gese et al. 1990). These techniques may allow for more accurate locations at the expense of very fine scale movements and may minimize habitat based sampling biases.

On going analyses are examining coyote movement patterns relative to topography, wolf urine scent grids, and human developments. The relationship of habitat use in high use areas (identified from movement patterns) to habitat use in core use areas (identified in home range analyses) is also being examined.

\section{Mortality}

The mortality rate of marked coyotes during this study was $31 \%(9 / 29)$. In the SAA (Fig 1) the mortality during the 1999-2000 research season was $47 \%$ (7/15). Human exploitation (shooting) caused $57 \%(4 / 7)$ of the observed mortalities, and $100 \%$ (7/7) of the observed mortalities were human caused. In the UNDA the mortality was $14 \%(2 / 14)$, human exploitation caused $50 \%(1 / 2)$ of the observed mortalities. The overall mortality due to human causes is $28 \%(8 / 29)$. According to Crabtree and Sheldon (1999) this population of coyotes could be considered moderately exploited $(25-49 \%$ annual human related mortality). The overall mortality rates in this study were slightly lower than the $37 \%$ annual mortality rate reported by Tzilkowski (1980) in Grand Teton National Park. This difference may be due to the reduction of cattle operations and changes in the human demographics of the Jackson Hole area over the past two decades.

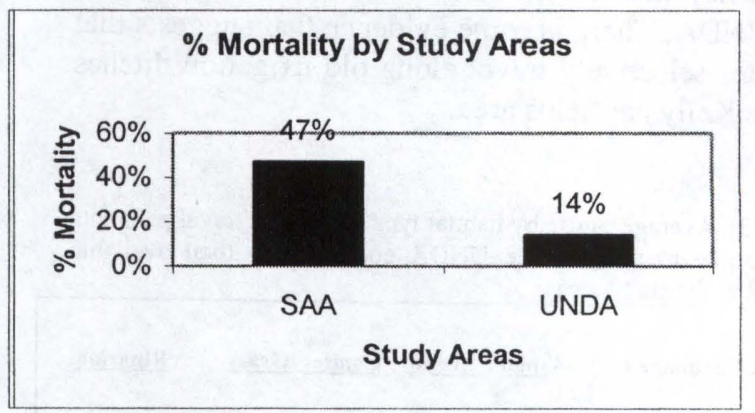

Fig 1

During this research a total of six male and three female coyotes died. Five males and two females died in the SAA, and one male and one female died in the UNDA. The gender breakdown for the total sample was 17 males and 12 females. Therefore, approximately $35 \%(6 / 17)$ of the males died, while only $25 \%$ (3/12) of the females died (Fig 2 ). This suggests that mortality rates are higher among male coyotes than female coyotes in this population.

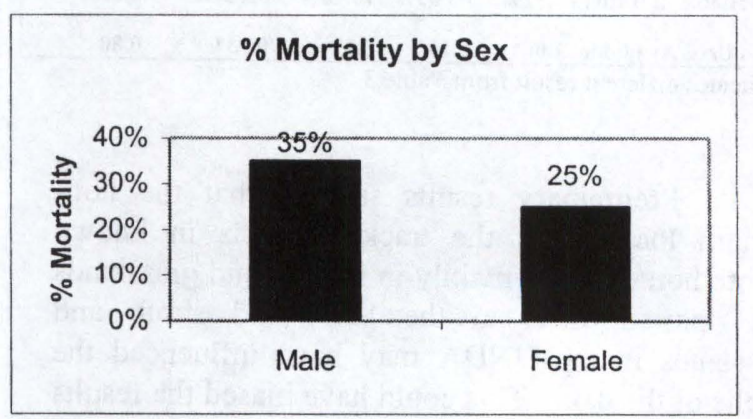

Fig 2

The overall leading cause of mortalities were gunshots $56 \%(5 / 9)$ followed by being hit by a car $33 \%(3 / 9)$, and being killed by coyotes $11 \%$ (1/9). Human caused mortalities made up $89 \%(8 / 9)$ of the overall mortalities. This data suggests that human caused mortalities are the most significant cause of mortality in this population, and that wolves are not currently a significant cause of mortality. 


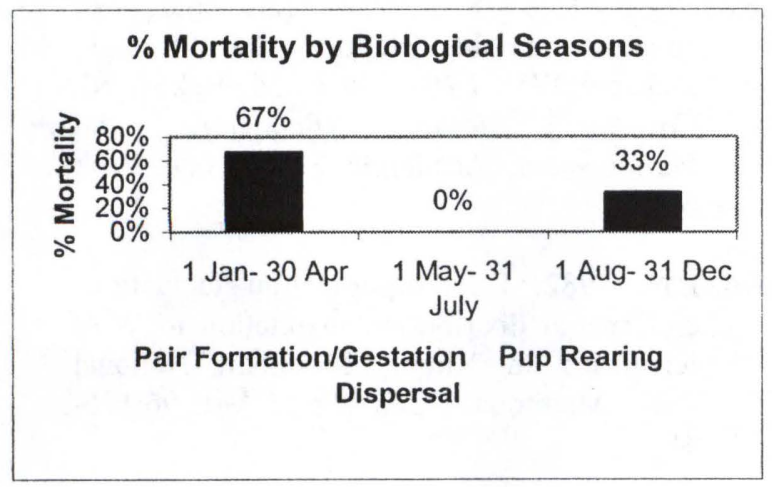

Fig 3

Overall, $67 \%$ of mortalities(Fig 3) occurred during the pair formation-gestation biological season (1 January to 30 April), $33 \%$ occurred during the dispersal season (1 August to 31 December), and none occurred during the pup-rearing season (1 May to 31 July). This pattern of seasonal mortality rates is somewhat atypical compared to most published accounts that indicate the majority of coyote mortality occurs during the dispersal season (Knowlton 1972). This may also be true of this population and could be determined if pup mortalities during the first year were measured. In adults however, it appears that the majority of the mortality does occur during the pair formation-gestation biological period. Since the majority of the observed mortalities resulted from gunshots (Fig 4 ) a nd most of the coyotes were shot during late winter-early spring in the SAA, it is possible that the high mortality during this season is a function of increased coyote control efforts during the calving season in ranching areas, which corresponds to the pair formation gestation season of coyotes.

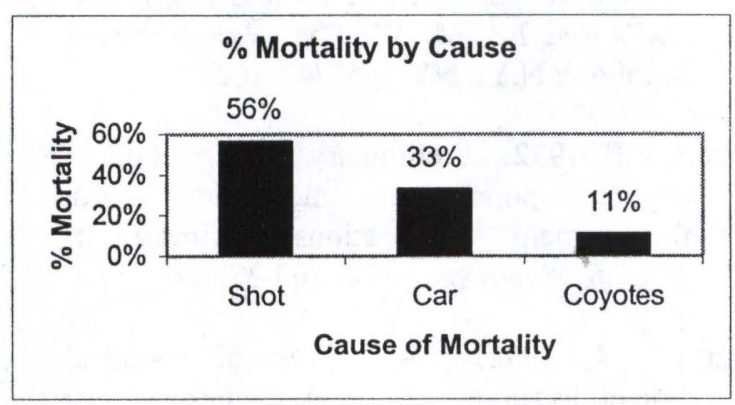

Fig 4

In terms of social type, the sample $(n=29)$ was made up of 18 residents and 11 transients. Approximately $64 \%$ (7/11) of the transients died, while only $11 \%(2 / 18)$ of the residents died (Fig 5). Human caused mortalities made up $100 \%(2 / 2)$ of the observed mortalities among resident coyotes and
$86 \%(6 / 7)$ of the observed mortalities among transient coyotes. This suggests that mortality rates among transient coyotes are higher than mortality rates among resident coyotes in this population, and that human caused mortality has the largest impact on both resident and transient coyotes.

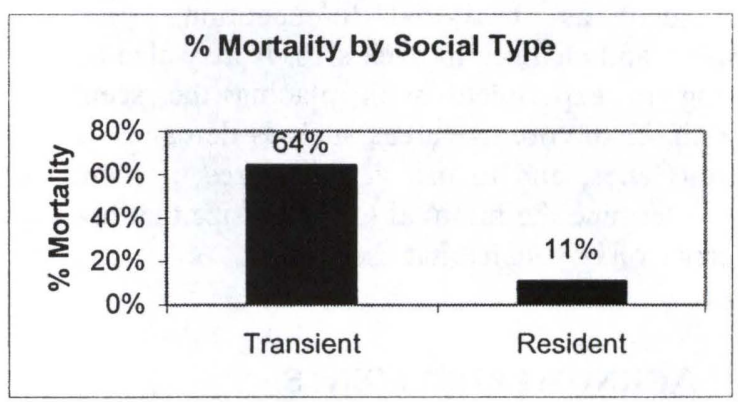

Fig 5

Preliminary investigation of the activity patterns suggests that there is no significant difference in activity (nocturnal vs. diurnal) between observed coyote mortalities $(n=9)$ and surviving coyotes $(n=20)$. Preliminary analyses of the spatial habitat use patterns of coyotes that died suggest that they used habitats closer to roads and human developments more frequently than coyotes that survived. Along with the fact that a majority of the mortalities were human caused, this data suggests that there may be a relationship between spatial habitat use relative to human development and coyote mortality. Further analyses will compare activity patterns between study areas, by gender and age to determine if these characteristics reveal any significant differences in mortality rates. Further analyses will also compare mortality data from McClennen (2000) and Wigglesworth (2000) with results from this research to determine average trends.

\section{Response to Wolf Urine}

Preliminary investigation of the data shows some shifts of experimental coyotes' home ranges within the UNDA, and no observable shift in the SAA $(n=10)$. There was no significant observed shift in the control coyotes in the UNDA or the SAA. In the UNDA on average $36 \%$ of the experimental coyote locations were within a 1000 meter buffer of their respective home range centers during the pretreatment time period and that only $5 \%$ of the experimental coyotes locations were within the 1000 meter buffers during the post-treatment time period. This suggests that there is some avoidance of the wolf urine scent grids by coyotes in the UNDA. 
There is a chance that the dimensions of the scent station grids were too small to have had a significant chance of exposing coyotes to the wolf urine, and that the observed shifts are in response to other unmeasured variables. Further investigations would be required to establish a baseline of the natural changes in coyote home ranges in response to factors such as territorial competition, prey availability, and changes in pack size. It may also be interesting to experiment with placing the scent stations closer to core use areas such as den sites or rendezvous sites, and to use various sizes of scent grids to determine the minimal effective size that has an influence on coyote habitat use.

\section{ACKNOWLEDGEMENTS}

The UW-NPS Research Center, the Teton Science School, Steve and Lisa Robertson, and the Wyoming Cooperative Fish and Wildlife Research Unit supported this research. I extend my thanks to Grand Teton National Park, the National Elk Refuge, and many private landowners in Jackson Hole who provided access and the necessary permits in order to conduct this research. Special thanks to the many volunteers, faculty, staff, and students of the Teton Science School who assisted in carrying out this research and supporting this project. Finally, I would like to thank my family for their interest and support, and Lisa Dardy for her support and friendship.

\section{Literature Cited}

Bekoff, M., and C.M. Wells. 1980. Social ecology of coyotes. Scientific American, April: 130148.

Berg, W.E., and R. A. Chesness. 1978. Ecology of coyotes in northern Minnesota. in Coyotes: biology, behavior, and management. ed M.Bekoff. Academic Press, NY. pp. 229247.

Bounds, D. L. 1994. Managing coyotes in U.S. National Parks: Human coyote interactions. Natural Areas Journal. 14:280-284.

Bowen, W. D. 1981. Variation in coyote social organization: The influence of prey size. Can. J. Zool. 59: 639-652.
Camenzind, F. J. 1978. Behavioral ecology of coyotes on the National Elk Refuge, Jackson, WY. P267-294 in M. Bekoff ed. Coyotes: Biology, Behavior, and Management. Academic Press. New York NY.

Carbyn, L.N. 1982. Coyote population fluctuations and spatial distribution in relation to Wolf territories in Riding Mountain National Park, Manitoba. Can. Field Nat. 96:176183.

Crabtree, R. L. and J. W. Sheldon. 1999. Coyotes and canid coexistence in Yellowstone. Pp 127-164 in Carnivores in ecosystems: the Yellowstone experience (T. W. Clark, S.C Minta, A.P. Curlee, and P.M. Kareiva, eds.). Yale University Press, New Haven, CT, USA.

Gese, E.M., O.J. Rongstad, and W.R. Mytton. 1988. Home range and habitat use of coyotes in southeastern Colorado. J ournal of Wildlife Management, 52: 640-646.

Gese, E.M., D. E. Anderson, and O. J. Rongstad. 1990. Determining home-range size of resident coyotes from point and sequential locations. Journal of Wildlife Management, 54(3): 501-506.

Gier, H. T. 1968. Coyotes in Kansas. Kansas State Coll. Agric. Exp. Stn. Bull. 393. 118pp.

Gier, H. T. 1975. Ecology and behavior of the coyote (Canis latrans). In: Wild Canids: Their Systematics, Behavioral Ecology, and Evolution. Ed. M. W. Fox. Van Nostrand Reinhold: N.Y., NY. pp. 247-262.

Knowlton, F.F. 1972. Preliminary interpretation of coyote population mechanics with management implications. Journal of Wildlife Management 36: 369-382.

Kreeger, T. J. 1992. A review of chemical immobilization of wild canids. in Proceedings of the Joint Conference of American Association of Zoo Veterinarians and American Wildlife Veterinarains. $d$ R. Junge Oakland, CA. 
Laundre, J. W., and B. L. Keller. 1981. Home range use by coyotes in Idaho. Animal Behavior, 29:449-461.

McClennen, N. 2000. The effect of agricultural and suburban development on the home Range size, activity, movement, and density of coyote (Canis latrans) in northwest Wyoming. M.S. Thesis, University of Wyoming, Laramie, WY. pp. 116.

Mech, L. D. 1966. The wolves of Isle Royale. USDI National Park Service, Fauna Series 7. 210 pp.,

Messier, F. and C. Barrette. 1982. The social system of the coyote (Canis latrans) in a forested habitat. Can. J. Zool. 60: 1743-1753.

Moore, T. D. and G. R. Parker. 1992. Colonization by the eastern coyote. Pages 23-28 in A. H. Boer, ed. Ecology and Management of the Eastern Coyote. Wildlife Research Unit, University of New Brunswick, Canada.

Murie, O.J. 1935. Food habits of the coyote in Jackson Hole, WY, United States Department of Agriculture Circular, 362: 124.

Nowak, R. M. 1978. Evolution and taxonomy of coyotes and related Canis. In Coyotes: Biology, behavior, and management. Edited by M. Bekoff. Academic Press, New York, NY. Pp. 229-247.
Servin, J., and C. Huxley. 1992. Immobilization of wild carnivores using ketamine and xilazine mixture. Veterinaria Mexico. 23(2): 135139.

Sheldon, J.W. 1992 Wild Dogs. Academic Press, San Diego, California.

Smith, G. J., J. R. Cary, a nd O. J. Rongstad. 1981. Sampling strategies for radio tracking Coyotes. Wildlife Society Bulletin 9: 88-93.

Toweil, D. E. and R. J. Anthony. 1988. Coyote food in a coniferous forest in Oregon. J. Wildl. Manage. 52: 507-512.

Tzilkowski, W. M. 1980. Patterns of radio-marked coyotes in Jackson Hole, Wyoming. Ph. D. Thesis. Univ. Mass., Amherst. 67 pp.

U.S. Census Bureau. 2000. States and County Quickfacts Teton County, Wyoming. [WWW Document]. URL http://quickfacts.census.gov/qfd/states/56/56 039.html

Weaver, J. L. 1977. Coyote food based relationships in Jackson Hole, Wyoming. M.S. Thesis, Utah State Univ., Logan, UT. 88 pp.

Wigglesworth, R., 2000. Habitat use, diet, social organization, and seroprevalence of diseases in coyotes (Canis latrans) in Grand Teton National Park and suburban/agricultural areas of northwest Wyoming. M.S. Thesis. University of Wyoming, Laramie, WY. pp. 124. 